\title{
Comparison of the pressure coefficient distributions obtained from EN 1991-1-4 to CFD analysis for hyperbolic paraboloid membrane structures
}

\author{
M. Van Craenenbroeck*, M. Mollaert ${ }^{\dagger}$ and L. De Laet ${ }^{\dagger}$ \\ * Department of Architectural Engineering (ARCH) \\ Vrije Universiteit Brussel \\ Pleinlaan 2, 1050 Elsene, Belgium \\ e-mail: maarten.van.craenenbroeck@vub.be,web page: https://vub.be/arch \\ ${ }^{\dagger}$ Department of Architectural Engineering $(\mathrm{ARCH})$ \\ Vrije Universiteit Brussel \\ Pleinlaan 2, 1050 Elsene, Belgium \\ web page: https://vub.be/arch
}

\begin{abstract}
Due to their low self weight, inherent form-active structural response, and unique geometries, membrane structures present some specific challenges to engineers. More specifically, predicting the magnitude and distribution of, as well as the structural response to wind loads on membrane structures requires considerable expertise.

Currently, there is no code available to provide a unified approach on how to deal with these highly dynamic loads on membrane structures similar to existing Eurocodes. With the ongoing work conducted by CEN/TC $248 \mathrm{WG} 4$ on the creation of such international standard, it has become important to not only better understand the nature of wind loads on membrane structures, but also understand the reasoning behind the existing Eurocode, EN 1991-1-4.
\end{abstract}

This paper explores the results of applying the methodology laid out by EN 1991-1-4 to a typical membrane structure geometry and compare the outcome to results obtained from a CFD/FSI simulation conducted in OpenFOAM.

Results clearly showed the limitations the existing Eurocode presents when it comes to accurately predicting pressure coefficient distributions on curved geometries, while at the same time including parts that are very relevant to membrane structures.

While it will not be possible to determine generalise $C_{p}$ distributions for every single membrane structure, and project-oriented approaches will still need to be conducted, the obtained results show that generalised distributions could be conceived for a number of "typical" membrane geometries bearing in mind the principles expressed in EN 1991-1-4.

\section{REFERENCES}

[1] CEN (European Committee for Standardisation), EN 1991-1-4:2005. Eurocode 1: Actions on Structures - Part 1-4: General actions - Wind actions (2005).

[2] J. Colliers, J. Degroote, M. Mollaert, and L. De Laet, "Mean pressure distributions over hyperbolic paraboloid roof and canopy structures with different shape parameters in a uniform flow with very small turbulence", Engineering Strutcures, 205, 0141-0296 (2019).

[3] F. Rizzo, G.A. Kopp, and G.F. Giaccu, "Investigation of wind-induced dynamics of a cable net roof with aerolastic wind tunnel tests", Engineering Structures, 229, 0141-0296 (2020). 